\title{
A symmetry breaking mechanism for selecting the speed of relativistic solitons*
}

\author{
Mariano Cadoni † Roberto De Leo $\ddagger$ \\ Dipartimento di Fisica, Università di Cagliari and INFN, Sezione di Cagliari \\ Cittadella Universitaria, 09042 Monserrato, Italy \\ Giuseppe Gaeta § \\ Dipartimento di Matematica, Università di Milano, \\ via Saldini 50, 20133 Milano, Italy
}

March 6, 2018

\begin{abstract}
We propose a mechanism for fixing the velocity of relativistic soliton based on the breaking of the Lorentz symmetry of the sine-Gordon (SG) model. The proposal is first elaborated for a molecular chain model, as the simple pendulum limit of a double pendulums chain. It is then generalized to a full class of two-dimensional field theories of the sine-Gordon type. From a phenomenological point of view, the mechanism allows one to select the speed of a SG soliton just by tuning elastic couplings constants and kinematical parameters. From a fundamental, field-theoretical point of view we show that the characterizing features of relativistic SG solitons (existence of conserved topological charges and stability) may be still preserved even if the Lorentz symmetry is broken and a soliton of a given speed is selected.
\end{abstract}

\section{Introduction}

In the past decades solitons have become a very useful concept in many areas of mathematical, theoretical and applied physics. Originally discovered as solitary waves solutions of non linear partial differential equations [1, 2, 3], solitons have become a generic paradigm of non linear physical phenomena [4, 5].

Of particular relevance, both from the theoretical and phenomenological point of view, are relativistic solitons, in particular topological two-dimensional (2D) sine-Gordon (SG) solitons (kinks) [6] , on which this paper will be mainly

\footnotetext{
${ }^{*}$ Work supported in part by the Italian MIUR under the program COFIN2004, as part of the PRIN project "Mathematical Models for DNA Dynamics $\left(M^{2} \times D^{2}\right)$ ".

†email: mariano.cadoni@ca.infn.it

¥email:roberto.deleo@ca.infn.it

§email:gaeta@mat.unimi.it
} 
focused. On the phenomenological side, SG solitons have wide range of applicability: non linear molecular [7] and DNA dynamics [8, 9, 10, 11, Josephson effect [12, ferromagnetic waves [13, 14, non linear optics [15, 16], superconductivity [17. and many others [18.

From a theoretical point of view SG solitons exhibit remarkable analogies with relativistic particles. They share with the latter the feature of being localized, non dispersive objects realizing the Lorentz symmetry. SG solitons are characterized by short range forces and they can interact without loosing their identities [19].

Moreover, topological SG solitons are stable and their existence is related to the discrete internal symmetries (and the existence of degenerate vacua) of the two-dimensional (2D) field theory of which they are classical solutions. Topology offers an elegant way of classifying solitons in terms of the homotopy group of the mappings between the vacua and the asymptotical field configurations [6].

The soliton-particle analogy has been very fruitful both for particle physics and quantum field theory. Starting with the pioneering work of Finkelstein and Misner [20], Rubinstein [6] and Skyrme 21], the analogy has found applications in various contexts such as S-Matrix formalism [22, current algebra effective Lagrangians [23], supersymmetric quantum mechanics [24], black hole physics [25. 26] just to mention a few of them.

Solitons have also became central in the study of integrable PDEs [27, 28, and in many geometrical matters (in particular connected with Field Theory), see e.g. [29].

A general feature of relativistic solitons and in particular of SG solitons, is the fact that the soliton speed $v$ is a free parameter, which can be fixed by choosing initial conditions and is bounded from above by a limiting value $c_{0}$. This is obvious in view of the Lorentz symmetry of the model: only the limiting upper bound (corresponding to the speed of light for a relativistic particle) of the soliton speed is fixed by the model, whereas $v$ can be changed applying a boost.

This degeneracy in $v$ is a necessary property of a relativistic field theory such as SG theory and appears rather natural in view of the soliton-particle analogy. However, in phenomenological situations where the experiments give a well-defined value for the propagation speed of the soliton it represents a loss of predictive power of the model. By modelling some non-linear dynamical process using a SG-like field theory very often it is crucial not only that solitary, non dispersive waves do exist but also that their velocity is at least of the same order of magnitude of that observed in experiments.

Thus, a selection mechanism for the soliton velocity should be very welcome from the phenomenological point of view. In this context an instructive example is represented by the use of solitons in the description of DNA non linear torsion dynamics. It is believed that kink solitons of the SG type play a crucial role in the DNA transcription process [8, 9]. In the past decades several models of DNA torsional dynamics have been proposed that allow for the existence of SG kink solitons ( see e.g [9, 10] and references therein). However, in most of them 
the soliton velocity is not fixed by the mode 1 , whereas the DNA transcription process, which the soliton propagation is assumed to model, occurs with a speed of a well-defined order of magnitude.

Although desirable from a phenomenological point of view, it is not very easy to implement a general selection mechanism for the soliton speed. The high sensibility of non linear systems to the initial conditions, practically rules out a selection mechanism based entirely on tuning initial conditions of the system.

On the other hand, one expects a speed selection mechanism to have a strong impact on the existence and on fundamental properties of SG solitons. In fact any velocity selection breaks the Lorentz symmetry of the model. It is not $a$ priori evident that after this breaking the theory will still allow for localized solutions with the features of the SG solitons such as stability and topological classification.

In this paper we will present and discuss in detail a velocity selection mechanism for SG topological solitons. Our starting point is a result we found in previous investigation of a composite model for DNA torsional dynamics, which is essentially a double chain of coupled pendulums [30, 31. We found that in the limiting case where one of the two coordinates describing the angular displacement of the pendulums is constrained to be identically zero, the continuum limit of the model has SG solitonic solutions with speed fixed in terms of the elastic and inertial parameters of the chain.

The possibility of selecting the speed of SG solitons by adding to the Lagrangian terms breaking the Lorentz symmetry has been first recognized in Ref. [32. A speed selection mechanism has been also found in the case of solitonic solutions of the non-linear Schrödinger equation 33. In particular, in Ref. 32 the authors consider a SG model modified by the addition of further kinetic and potential terms. In this paper we consider instead a two fields generalization of the usual SG model. Moreover the breaking of the Lorentz symmetry, which is responsible for the fixing of the soliton speed is rather subtle. It is generated by the presence of two (instead of one) upper limit speeds for the propagation of waves.

The plan of the paper is as follows. We will first review the results of Ref. 30 and reformulate them as the simple pendulum limit of a double pendulums molecular chain model (Section 2). We will show that the selection mechanism is related to the existence of a conditionally conserved quantity, a conservation law that holds only when the system is constrained in a limited region of its phase space, i.e. to a region of the reduced one-dimensional (1D) equations of motion describing propagating waves (Section 3). We will then pass to consider the most general model for which the selection speed mechanism does work. We will show that the general model can be formulated as a two fields generalization of 2D SG field theory (Section 4). We will consider a simplified, minimal version of the model, which has the nice feature of being explicitly solvable in its general

\footnotetext{
${ }^{1}$ The only exception is the model of Ref. [30, where the selection mechanism discussed in this paper was first observed but not identified in its generality.
} 
form (Section 5). The breaking of the Lorentz symmetry is discussed and it is shown that it is related to the presence of two (instead of one) upper bound limiting speeds (Section 6). The discrete symmetries of the model are discussed and used for the topological classification of the kinks (Section 7). The stability of our solitonic solutions are also investigate. In particular we show that the Lorentz symmetry breaking term acts as damping term for linear perturbation near the soliton (Section 8). Finally we state our conclusions (Section 9).

\section{Soliton speed selection in molecular chain models}

Let us start from the double pendulums chain model of Ref. 30] modeling DNA torsional dynamics. The two DNA helices are modeled by two chains of double pendulums. At each, equally spaced with distance $\delta$, site of the two chains there is a first pendulum, which is a disk radius $R$ and momentum of inertia $I$. Attached to the border of the disk there is second pendulum of length $r$ and mass $m$. The rotation angles of the two pendulums with respect to the equilibrium positions are denoted respectively by $\theta_{i}^{a}, \varphi_{i}^{a}$, where the index $a=1,2$ refers to elements in the two chains and $i \in \mathbf{Z}$ identifies the site in the chain and $\theta_{i}^{a}, \varphi_{i}^{a} \in S^{1}$. The pendulums interact with nearest-neighbor interactions of three types: stacking (coupling between successive pendulums on the same chain), pairing (coupling between pendulums on the same site but opposite chain) and torsional (coupling between successive disks on the same chain), characterized by coupling (elastic) constants given respectively by $K_{s}, K_{p}, K_{t}$. Moreover, the second pendulum is not free to swing through a full circle but it is instead constrained to stay in a range $\varphi_{i}^{a} \leq \varphi_{0}<2 \pi$. This constraint can be modelled by adding a confining potential $V_{c}(\varphi)$, which has the effect of limiting de facto the excursion of the $\varphi$ angles; see Ref. [30, 31] for details.

As here we are interested not in the details of the model but in its general behavior, we will make, with respect to Ref. [30, 31, a number of simplifying assumptions. First we will consider from the beginning only symmetric solutions, $\theta_{i}^{1}=\theta_{i}^{2}:=\theta_{i}, \varphi_{i}^{1}=\varphi_{i}^{2}:=\varphi_{i}$. This position reduces the degrees of freedom from four to two per site. Second, to reduce the number of kinematical parameters we will set $R=r$. Under these simplifying assumptions, in the continuum $(\delta \rightarrow 0)$ limit the dynamics of the model is described by a 2D field theory with Lagrangian density

$$
\begin{aligned}
\mathcal{L} & =\frac{1}{2}\left\{I \theta_{t}^{2}-\omega_{t} \theta_{x}^{2}+r^{2}\left[m \theta_{t}^{2}-\omega_{s} \theta_{x}^{2}+2 \cos (\varphi)\left(m \theta_{t}\left(\varphi_{t}+\theta_{t}\right)+\right.\right.\right. \\
& \left.\left.\left.-\omega_{s} \theta_{x}\left(\varphi_{x}+\theta_{x}\right)\right)+m\left(\varphi_{t}+\theta_{t}\right)^{2}-\omega_{s}\left(\varphi_{x}+\theta_{x}\right)^{2}\right]\right\}+ \\
& \left.+4 r^{2} K_{p}\left(\cos \theta+\cos (\varphi+\theta)-\frac{1}{2} \cos (\varphi)-\frac{3}{2}\right)\right)-V_{c}(\varphi),
\end{aligned}
$$

$x$ is the spatial coordinate along the chain, the fields $\theta(t, x)$ and $\varphi(t, x)$ represent the angular coordinate $\theta_{i}$ and $\varphi_{i}$ in the continuum limit, the indices $x$ and 
$t$ denote partial derivation and $\omega_{t}=K_{t} \delta^{2}, \omega_{s}=K_{s} \delta^{2}$. We will use in the Lagrangian (11) a rather generic confining potential $V_{c}$. We will only assume that $V_{c}(\varphi)$ is an even function of $\varphi$, which has a minimum at $\varphi=0$. Moreover, we can take without loss of generality $V_{c}(0)=0$

At this point the interpretation of the Lagrangian (11) as modelling the DNA torsional dynamics is not anymore compulsory, nor so relevant. We can as well consider the Lagrangian as describing the continuum limit of the torsional dynamics of a single molecular chain made of two pendulums. The pairing interaction for the DNA double chain becomes an external potential for the pendulums: $V=-4 r^{2} K_{p}\left(\cos \theta+\cos (\varphi+\theta)-\frac{1}{2} \cos \varphi-3 / 2\right)$, whereas the pairing and torsional interaction generate in the Lagrangian (11) the gradient ( $x$-derivative) terms.

Let us consider travelling waves solutions of the field theory (10), i.e solutions depending only on $z=x+v t$ with fixed speed $v: \theta=\theta(z), \varphi=\varphi(z)$. With this position the field equations stemming from (11) become

$$
\begin{aligned}
& \mu \varphi^{\prime \prime}+\mu(1+\cos \varphi) \theta^{\prime \prime}= \\
& \quad=-4 K_{p} \sin (\varphi+\theta)-\mu \sin (\varphi)\left(\theta^{\prime}\right)^{2}+2 K_{p} \sin (\varphi)-\frac{\partial V_{c}}{\partial \varphi} ; \\
& \mu(1+\cos \varphi) \varphi^{\prime \prime}+\left[\left(J / r^{2}\right)+2 \mu(1+\cos \varphi)\right] \theta^{\prime \prime}= \\
& \quad=-4 K_{p}(\sin \theta+\sin (\varphi+\theta))+\mu \sin (\varphi)\left[\left(\varphi^{\prime}\right)^{2}+2 \varphi^{\prime} \theta^{\prime}\right],
\end{aligned}
$$

where the prime denotes derivatives with respect to $z$ and following the notation of Ref. [30] we define

$$
\mu:=\left(m v^{2}-\omega_{s}\right), \quad J:=\left(I v^{2}-\omega_{t}\right) .
$$

In general the system (2) cannot be solved analytically in closed form. One has to resort to numerical calculations in order to show that the system admits solitonic solutions [30. However, in Ref. [30] it was shown that one can find a solitonic solution with fixed speed, just by freezing the angle $\varphi$, i.e by setting $\varphi=0$. We note that if we force $\varphi(z)=0$, we are actually considering a chain of simple pendulums, i.e. a sine-Gordon equation. This constraint can be accommodated in our setting in a dynamical way, by acting on the confining potential $V_{c}$ : this should be made stronger and stronger and the maximum angle $\varphi_{0}$ smaller and smaller. In the limit $\varphi_{0} \rightarrow 0^{+}$and $\left(\partial^{2} V_{c} / \partial \varphi^{2}\right)(0) \rightarrow+\infty$, we expect to recover the solitons of the sine-Gordon equation.

Setting $\varphi=0$ and using $\left(\partial V_{c} / \partial \varphi\right)(0)=0$, the system (2) is equivalent to the SG field equation

$$
\mu \theta^{\prime \prime}=-2 K_{p} \sin \theta
$$

with the compatibility condition between the two equations of the system (2) given by

$$
J=0 .
$$

In principle one could consider a generic constraint $\varphi=q \theta$, where $q$ is a proportionality constant. However, it is not difficult to realize that for $q \neq 0$ such a constraint, when used in Eqs. (2), will lead to two different determinations of $\theta$ making the two resulting equations incompatible. 
Eq. (44) has to be integrated with the usual boundary conditions for kinks $\theta( \pm \infty)=2 n_{ \pm} \pi, \theta^{\prime}( \pm \infty)=0$ with $n_{ \pm} \in \mathbf{Z}$. Here $n=n_{+}-n_{-}$is the kink winding number. For $n=1 \mathrm{Eq}$. (4) can be easily integrated to give the kink

$$
\theta_{0}=4 \arctan \left[e^{\mathcal{K} z}\right], \quad \varphi=0, \quad \mathcal{K}=\sqrt{\frac{2 K_{p}}{|\mu|}},
$$

whereas the compatibility condition (5) fixes the speed of propagation of the soliton to the speed $c_{t}$ of the transverse sound waves supported by the elastic torsional forces acting on the disk

$$
v=c_{t}=\sqrt{\omega_{t} / I}
$$

Moreover, because the soliton exist only for $\mu<0$, the soliton speed is bounded from above by the speed $c_{s}$ of transverse sound waves supported by the elastic stacking forces acting on the pendulum, i.e.

$$
v \leq c_{s}=\sqrt{\omega_{s} / m}
$$

In view of Eq. (7), this implies the following constraint on the stacking, torsional coupling constants and kinematical parameters of the system:

$$
\frac{K_{t}}{I}<\frac{K_{s}}{m}
$$

The condition $\varphi=0$ fixes the speeds of the soliton to the value (7) also for kinks with winding numbers $n>1$. In fact, in Sect. 7 we will show that the fixed speed solitonic solutions of Eq. (4) allow for the same topological classification of the usual SG solitons.

The selection mechanism for the soliton speed discussed above represents a very nice and simple way to produce SG solitons with a given speed in double pendulums molecular chains. To select the soliton speed one just needs to tune the torsional and stacking coupling constants and the kinematical parameters of the chain such that Eqs. (7) and (9) are satisfied. Acting on the confining potential $V_{c}$, making it stronger and stronger, one obtains the single pendulum limit of the double pendulums chain. The angle is frozen to $\varphi=0$ and a SG soliton with a speed equal to that of the transverse sound waves supported by the torsional forces acting on the disk is selected. Notice that the above described mechanism can be obviously used to devise and realize non-linear media where solitons propagate at a given fixed speed.

Although very simple the mechanism we are proposing raises a number of questions. How is our mechanism related to fundamental features of the SG system such as integrability, Lorentz symmetry and topological classification? Can our mechanism be generalized to a broad class of models or is it just an artifact of the peculiar couplings present in the Lagrangian (1)? Is the SG soliton we are selecting stable? We will try to answer these questions in the following sections. 


\section{Integrability and conditionally conserved quantities}

The simple (one single degree of freedom) SG system (4) is integrable, in the sense that the initial value problem can always be solved analytically in closed form. Moreover, this integrability is related to the existence of a conserved quantity (a first integral of the equations of motion), which can be easily identified as the energy of the system.

On the other hand, the (two degrees of freedom) system (2) in general allows for only one conserved quantity - the energy - and it is not integrable in the sense used above. Nonetheless, it can be solved analytically in closed form and reduces to the SG system (4) if we limit ourselves to consider only $\varphi=0$ solutions. One is therefore led to search for a conditionally conserved quantity (a discussion of this weak form of integrability can be found in Ref. [34]). That is, a quantity that in general is not conserved by the dynamical evolution of the system, but which becomes conserved when the dynamics is restricted to the subspace $\varphi=0$.

To this aim, let us first note that the equations of motion (2) describe a dynamical system with two degrees of freedom, with the coordinate $z$ playing the role of the time. The equation of motion can be derived from the Lagrangian $L=T-V$,

$$
\begin{aligned}
L & =\frac{1}{2} J\left(\theta^{\prime}\right)^{2}+\frac{\mu r^{2}}{2}\left[\left(\theta^{\prime}\right)^{2}+2 \cos (\varphi)\left(\left(\theta^{\prime}\right)^{2}+\theta^{\prime} \varphi^{\prime}\right)+\left(\theta^{\prime}+\varphi^{\prime}\right)^{2}\right] \\
& -2 K_{p} r^{2}[\cos \varphi-2 \cos (\theta+\varphi)-2 \cos \theta]-V_{c}(\varphi) .
\end{aligned}
$$

With a little algebra we obtain from the system (2) the equation,

$$
\frac{d}{d z} P\left(\theta^{\prime}, \varphi^{\prime}, \varphi\right)=\mathcal{F}\left(\theta, \varphi,\left(\theta^{\prime}\right)^{2},\left(\varphi^{\prime}\right)^{2}\right),
$$

where $\mathcal{F}$ is given by

$$
\mathcal{F}=2 r^{2}\left[2 K_{p}(\sin (\theta+\varphi)-\sin \theta-\sin \varphi)+\mu \sin \varphi\left(\left(\theta^{\prime}\right)^{2}+\varphi^{\prime} \theta^{\prime}\right)\right]+2 \frac{\partial V_{c}}{\partial \varphi} .
$$

and $P$ is linear in the momenta $P_{\theta}=\delta L / \delta \theta^{\prime}, \quad P_{\varphi}=\delta L / \delta \varphi^{\prime}$ :

$$
P=P_{\theta}-2 P_{\varphi}=J \theta^{\prime}+s(\varphi) \mu \varphi^{\prime}, \quad s=r^{2}(\cos \varphi-1) .
$$

Notice that the coefficient $s$, its derivative with respect to $\varphi$ and $\mathcal{F}$ vanish when

evaluated on $\varphi=0$. The physical interpretation of Eq. (11) is very simple: this is just Newton's second law with $P$ playing the role of the impulse and $\mathcal{F}$ that of the force.

The equations of motion (2) can be now rewritten in the equivalent form

$$
\begin{aligned}
\mu \varphi^{\prime \prime}+\mu(1+\cos \varphi) \theta^{\prime \prime} & =-4 K_{p} \sin (\varphi+\theta)-\mu \sin (\varphi)\left(\theta^{\prime}\right)^{2}+2 K_{p} \sin (\varphi) \\
P^{\prime} & =\mathcal{F} .
\end{aligned}
$$


In general the momentum $P$ is not conserved, but becomes conserved when $\mathcal{F}=0$. This can be achieved if we restrict ourself to consider only the solutions of Eqs. (14) that satisfy $\varphi=0$. Taking into account that $\mathcal{F}(0)=s(0)=s^{\prime}(0)=0$ Eq. (11) gives a conservation law for

$$
P(\varphi=0):=H=J \theta^{\prime}
$$

Thus, $H$ is the conditionally conserved quantity we have been searching for.

It can be interpreted has an "effective" angular momentum of the disk. In fact $J$ has two contributions, see Eqs. (3). The first comes from the geometrical momentum of inertia of the disk, the second has opposite sign and comes from $K_{t}$, the elastic forces acting on the disk.

For $\varphi=0$, Eqs. (14) reduce to

$$
\mu \theta^{\prime \prime}=-2 K_{p} \sin \theta, \quad \frac{d H}{d z}=0 .
$$

For $J \neq 0$ the previous equations do not admit solitonic solutions (actually the only solution is the trivial one $\theta=0$ ). Solitonic solutions exist only for $J=0$, when the conservation law is trivialized. In this case Eqs. (16) coincide with Eq. (4), (5), which give the fixed speed soliton solution (6).

Thus the physical mechanism behind the fixing of the soliton speed is the following. When restricted to the subspace of solutions $\varphi=0$ the system has a conservation law, which is not compatible with the existence of solitonic excitations. The requirement of existence of solitons implies a trivialization of the conservation equation, which in turn fixes the soliton speed. From this point of view the condition $J=0$ can be seen as a sort of resonance condition: both the force and the "effective" mass in equation (11) must be zero.

\section{The general model as a 2D SG-like field theory}

The mechanism for selecting the soliton speed described in the previous sections for the molecular chain model (10) is rather generic. As we have shown in the previous section, the speed selecting mechanism is related to the existence of a conditionally conserved quantity and it is rather independent from the specific form of the interactions characterizing the model. Therefore, it will be present if the system satisfies some general conditions.

\subsection{The general speed selection mechanism in molecular chains}

Below is a list of the main ingredients that are needed for the mechanism to work in the case of a molecular chain. We need a chain (array) of identical mechanical systems with the following features:

1. The system must have at least two degrees of freedom $(X, Y)$ characterized by two masses (or momenta of inertia) $m, M$ with $m \neq M$; 
2. There are at least two types of interactions: $a$ ) An elastic force (coupling constant $K_{t}$ ) originated by the interaction between neighboring sites on the chain; b) A non linear external force (coupling constant $K_{p}$ ) acting on the single site;

3. There is a confining potential $V_{c}$ that limits the range of variation of one degree of freedom (e.g $Y$ ) and allows to freeze $Y$. That is by making $V_{c}$ stronger and stronger we perform the dynamical reduction from two to one single degree of freedom $X$;

4. Freezing the degree of freedom $Y$ we have both a conservation law for the momenta conjugate to $X$ and solitonic solutions for $X$.

If the conditions above are satisfied, then the mechanism described in the previous sections will work. In particular, considering the continuum limit of the chain and travelling wave solutions $X(z)=X(x+v t), Y(z)=Y(x+v t)$ we get a system of two differential equations which describe a mechanical system with two degrees of freedom with effective masses 2

$$
m_{\mathrm{eff}}=v^{2} m-K_{t} \delta^{2}, \quad M_{\mathrm{eff}}=v^{2} M-K_{t} \delta^{2} .
$$

As the conservation of the momentum conjugate to $X$ (for $Y$ frozen), $P_{X}=$ $M_{\text {eff }} d X / d z$ is not compatible with the existence of solitons, this will require $M_{\text {eff }}=0$, which will fix the soliton speed in terms of transverse phonon speed of the chain,

$$
v=\delta \sqrt{\frac{K_{t}}{M}}
$$

Moreover, $m_{\mathrm{eff}}$ is the analogous of the parameter $\mu$ in Eqs. (3) and (4), hence the soliton will exist only for $m_{\mathrm{eff}}<0$, implying $v<\delta \sqrt{K_{t} / m}$. Consistency of these equations requires $M>m$.

\subsection{Speed selection in field theory}

The explicit construction of models satisfying the previous conditions becomes much simpler if we consider them directly at the field theoretical, Lagrangian level, as generalization of the SG system, rather then as originated from a molecular chain. The main advantage of this approach is that the speed selection mechanism becomes a general, field theoretical, effect not necessarily originated from the continuum limit of some discrete model. In the rest of this paper we will follow this approach and we will make no more reference to molecular chain models.

To write down the Lagrangian for a general class of field theories that exhibits the speed selection effect, we first redefine the field $\varphi$ in the Lagrangian density

\footnotetext{
${ }^{2}$ Depending on the particular model one is considering, the equations below may also assume a more general form $m_{\text {eff }}=v^{2} m-f\left(r_{i}\right) K_{t} \delta^{2}$ (and similarly for $M_{\text {eff }}$ ), where $f\left(r_{i}\right)$ is a function of the geometrical parameters $r_{i}$ characterizing the model.
} 
(11). Defining $\varphi=\Phi-\theta$ the Lagrangian (11) becomes

$$
\begin{aligned}
& \mathcal{L}=\frac{1}{2}\left\{I \theta_{t}^{2}-\omega_{t} \theta_{x}^{2}+r^{2}\left[m \theta_{t}^{2}-\omega_{s} \theta_{x}^{2}+2 \cos (\Phi-\theta)\left(m \theta_{t} \Phi_{t}-\omega_{s} \theta_{x} \Phi_{x}\right)+\right.\right. \\
& \left.\left.+m \Phi_{t}^{2}-\omega_{s} \Phi_{x}^{2}+8 K_{p}\left(\cos \theta+\cos \Phi-\frac{1}{2} \cos (\Phi-\theta)-\frac{3}{2}\right)\right]\right\}-V_{c}(\Phi-\theta) .
\end{aligned}
$$

In this new parametrization, the fixed speed soliton solution is given by $\Phi=$ $\theta=\theta_{0}$, with $\theta_{0}$ given by Eq. (6).

Solitons are localized excitations of finite energy. The energy of the solution (6) can be calculated from the Hamiltonian density. From the Lagrangian (19) one can easily obtain the Hamiltonian density

$$
\begin{aligned}
& \mathcal{H}=\frac{1}{2}\left\{I \theta_{t}^{2}+\omega_{t} \theta_{x}^{2}+r^{2}\left[m \theta_{t}^{2}+\omega_{s} \theta_{x}^{2}+2 \cos (\Phi-\theta)\left(m \theta_{t} \Phi_{t}+\omega_{s} \theta_{x} \Phi_{x}\right)+\right.\right. \\
& \left.\left.+m \Phi_{t}^{2}+\omega_{s} \Phi_{x}^{2}-8 K_{p}\left(\cos \theta+\cos \Phi-\frac{1}{2} \cos (\Phi-\theta)-\frac{3}{2}\right)\right]\right\}+V_{c}(\Phi-\theta) .
\end{aligned}
$$

The constant field configuration of minimal energy (vacua) can be obtained by minimizing the potential term in Eq. (20). They are given by $\theta=\Phi=2 n \pi, n \in$ $\mathbf{Z}$ and have zero energy. The energy of the soliton (6), connecting vacua with $n_{+}=n_{-}+1$, is

$$
H=\int_{-\infty}^{\infty} \mathcal{H} d x=16 r^{2} K_{p} \frac{\omega_{t}+8 r^{2} \omega_{s}}{\omega_{s}-(m / I) \omega_{t}} .
$$

As expected the energy of the soliton (measured with respect to that of the vacuum) is finite and positive in the range of existence of the soliton.

It is useful, in particular in view of the discussion about the Lorentz symmetry of the theory, to write the Lagrangian using a Minkowski spacetime notation. Introducing a spacetime metric of signature $(1,-1)$, the two fundamental speeds $c_{t}, c_{s}$ (we assume $c_{t}<c_{s}$ ) given by Eqs (8) and respectively (7), and the derivative operators

$$
\hat{\partial}_{\nu}=\left(\frac{\partial}{c_{t} \partial t}, \frac{\partial}{\partial x}\right), \quad \partial_{\nu}=\left(\frac{\partial}{c_{s} \partial t}, \frac{\partial}{\partial x}\right),
$$

the Lagrangian (19) takes the form

$$
\begin{array}{r}
\mathcal{L}=\frac{\omega_{t}}{2} \hat{\partial}_{\nu} \theta \hat{\partial}^{\nu} \theta+\frac{r^{2} \omega_{s}}{2}\left(\partial_{\nu} \theta \partial^{\nu} \theta+2 \cos (\Phi-\theta) \partial_{\nu} \theta \partial^{\nu} \Phi+\partial_{\nu} \Phi \partial^{\nu} \Phi\right) \\
+4 r^{2} K_{p}\left(\cos \theta+\cos \Phi-\frac{1}{2} \cos (\Phi-\theta)-\frac{3}{2}\right)-V_{c}(\Phi-\theta) .
\end{array}
$$

Generalizing the Lagrangian (23) one obtains a class of models admitting solitonic solutions with fixed speed.

The models have the form of a sine-Gordon-like coupled system of two scalar fields and will be functionally parametrized by two coupling functions $F, G$. In 
the Minkowski spacetime notation the Lagrangian is given by

$$
\begin{aligned}
\mathcal{L}_{g} & =\frac{a}{2} \hat{\partial}_{\nu} \theta \hat{\partial}^{\nu} \theta+\frac{b}{2} \partial_{\nu} \theta \partial^{\nu} \theta+F(\Phi-\theta) \partial_{\nu} \theta \partial^{\nu} \Phi+ \\
& +\frac{b}{2} \partial_{\nu} \Phi \partial^{\nu} \Phi+K(\cos \theta+\cos \Phi-2)+G(\Phi-\theta)
\end{aligned}
$$

where $a, b, K$ are positive constant parameters and the coupling functions $F(\Phi-$ $\theta$ ) and $G(\Phi-\theta)$ are arbitrary even functions of $\Phi-\theta$ and $G$ satisfies $G(0)=0$. Considering only travelling wave solutions $\Phi(z), \theta(z)$ (with $z=x+v t$ ) we obtain the $1 \mathrm{D}$ reduced Lagrangian which is the generalization of the one given in (10):

$$
L=\frac{1}{2} \widetilde{J}\left(\theta^{\prime}\right)^{2}+\frac{\widetilde{\mu}}{2}\left[\left(\theta^{\prime}\right)^{2}+2 F \theta^{\prime} \Phi^{\prime}+\left(\Phi^{\prime}\right)^{2}\right]+K[\cos \Phi+\cos (\theta)-2]+G,
$$

where

$$
\widetilde{J}=a\left(v^{2} / c_{t}^{2}-1\right), \quad \widetilde{\mu}=b\left(v^{2} / c_{s}^{2}-1\right) .
$$

Taking into account that because of the parity of the coupling functions $F$ and $G$ we have $F^{\prime}(0)=G^{\prime}(0)=0$, it is not difficult to show that for $\Phi=\theta$ the equation of motion stemming from the action(25) admits the SG soliton (6) travelling at speed $v=c_{t}<c_{s}$.

A further generalization, which we will not investigate in detail in this paper, can be obtained considering generically a self-interacting scalar field $\Theta$ in $2 \mathrm{D}$ Minkowski spacetime,

$$
\mathcal{L}=\frac{1}{2} \partial_{\nu} \Theta \partial^{\nu} \Theta-V(\Theta)
$$

whose potential $V$ is such that field equations for $\Theta$ admit relativistic soliton solutions (apart from the SG system, the model contains as a particular case e.g the $\varphi^{4}$ interaction model). A general Lagrangian describing two interacting scalar fields $\Theta, \Sigma$ for which the speed selection mechanism for the soliton applies is given by

$$
\begin{aligned}
\mathcal{L}_{g} & =\frac{a}{2} \hat{\partial}_{\nu} \Theta \hat{\partial}^{\nu} \Theta+\frac{b}{2} \partial_{\nu} \Theta \partial^{\nu} \Theta+F(\Theta-\Sigma) \partial_{\nu} \Theta \partial^{\nu} \Sigma+\frac{b}{2} \partial_{\nu} \Sigma \partial^{\nu} \Sigma \\
& -V(\Theta)-V(\Sigma)-G(\Theta-\Sigma)
\end{aligned}
$$

where as above $F(\Theta-\Sigma)$ and $G(\Theta-\Sigma)$ are arbitrary even functions of $(\Theta-\Sigma)$.

\section{Minimal model}

The Lagrangian (24) corresponds to a system of two coupled sine-Gordon models. It is of interest to see if the properties concerning the selection of the soliton speed hold also when the interaction terms are switched off, i.e when the coupling functions $F, G$ are identically zero. Moreover in the completely non-interacting case, the system will be integrable and we will have complete control on the general solutions. 
For $F=G=0$ the Lagrangian (24) becomes

$$
\mathcal{L}_{m}=\frac{a}{2} \hat{\partial}_{\nu} \theta \hat{\partial}^{\nu} \theta+\frac{b}{2} \partial_{\nu} \theta \partial^{\nu} \theta+\frac{b}{2} \partial_{\nu} \Phi \partial^{\nu} \Phi+K(\cos \theta+\cos \Phi-2) .
$$

The field equations describe a decoupled system for $\theta$ and $\Phi$,

$$
\frac{a}{b} \hat{\partial}_{\nu} \hat{\partial}^{\nu} \theta+\partial_{\nu} \partial^{\nu} \theta=-\frac{K}{b} \sin \theta, \quad \partial_{\nu} \partial^{\nu} \Phi=-\frac{K}{b} \sin \Phi .
$$

The theory contains two upper bound speeds, $c_{s}, c_{m}$ for the propagation of respectively $\Phi$ - and $\theta$-waves. $c_{m}$ is given by

$$
c_{m}=c_{t} c_{s} \sqrt{\frac{a+b}{a c_{s}^{2}+b c_{t}^{2}}} .
$$

Notice that $c_{s}>c_{t}$ implies necessarily also $c_{s}>c_{m}$.

Because the two scalars are decoupled we can look for traveling wave solutions of different speeds $v, \bar{v}$ for the two fields: $\Phi(z)=\Phi(x+v t), \theta(\bar{z})=\theta(x+\bar{v} t)$. In this way we we end up with a decoupled system of differential equations,

$$
\left(\frac{v^{2}}{c_{s}^{2}}-1\right) \Phi^{\prime \prime}=-\frac{K}{b} \sin \Phi, \quad\left[\frac{a}{b}\left(\frac{\bar{v}^{2}}{c_{t}^{2}}-1\right)+\left(\frac{\bar{v}^{2}}{c_{s}^{2}}-1\right)\right] \ddot{\theta}=-\frac{K}{b} \sin \theta,
$$

where the prime and the dot denote derivation with respect to $z$ and $\bar{z}$ respectively. The general solution of the previous system is given by two sine-Gordon solitons of type (6) with speeds $v \leq c_{s}$ and $\bar{v}<c_{m}<c_{s}$, respectively for the fields $\Phi$ and $\theta$. Only imposing $\theta=\Phi$ we have velocity selection. In fact $\theta=\Phi$ implies $v=\bar{v}=c_{t}$ Notice that the converse is not true. $v=c_{t}$ does not necessarily imply $\theta=\Phi$ or $v=\bar{v}$. For $v=c_{t}$ and $\theta \neq \Phi$ we will have solitons in $\theta$ and $\Phi$ that differ in speed or, if the speed is the same, in the coordinate of the center of the soliton ( we will have two identical solitons with non-zero relative phase).

\section{Lorentz Symmetry}

It is well known that usual SG field theory, described by the $2 \mathrm{D}$ action

$$
S=\int\left[\frac{\left(\varphi_{t}\right)^{2}}{2 c_{0}^{2}}-\frac{1}{2}\left(\varphi_{x}\right)^{2}+\omega_{0}(\cos \varphi-1)\right] d x d t
$$

is invariant under 2D Lorentz transformations (boosts)

$$
x=\gamma\left(x^{\prime}+v t^{\prime}\right), \quad t=\gamma\left(t^{\prime}+\frac{v}{c_{0}^{2}} x^{\prime}\right), \quad \gamma=\frac{1}{\sqrt{1-\frac{v^{2}}{c_{0}^{2}}}},
$$

where $c_{0}$ plays the role of the speed of light in vacuum. The Lorentz symmetry of the action (32) is essential for establishing the soliton/relativistic particle 
analogy and allows one to use for SG solitons the concepts of relativistic kinematics. For instance, Lorentz symmetry allows us to generate, using a boost (33), a SG soliton propagating with speed $v$

$$
\varphi(x+v t)=4 \arctan \left[e^{\omega_{0} \gamma(x+v t)}\right]
$$

from the static solution $\varphi_{0}(x)=4 \arctan \left[e^{\omega_{0} x}\right]$. Another consequence of the Lorentz symmetry of the theory is the existence of a maximum propagation speed, $v_{\max }=c_{0}$ for the solitons. To make the Lorentz symmetry more evident we might write the action (32) using the Minkowskian notation of Eqs. (22).

On the other hand, the two-fields generalized SG theory (24) we are discussing in this paper is not Lorentz invariant. This is due the fact that in the Lagrangian (24) appear not a single upper bound speed $c_{0}$ but two of them $c_{s}, c_{m}$. It follows that we have the choice of defining the boosts (33) either in terms of $c_{s}$ or $c_{m}$. In the first case the Lorentz symmetry is broken by the kinetic term proportional to $a$, in the second case by the kinetic terms proportional to $b$ in the action (24). Because $c_{m}<c_{s}$ it is natural to consider boosts of the first kind, so that it is the $a$-term that breaks the Lorentz symmetry.

From the physical point of view, the breaking of the Lorentz symmetry can be easily understood if one realizes that our system can be thought as a medium with two different speeds for the propagation of sound waves.

It is obvious that the speed selection effect described in previous sections is strongly related to breaking of the Lorentz symmetry. In fact if in Eq. (24) we set $a=0$ we have no selection for the speed of the soliton and Lorentz symmetry is not broken. Let us therefore investigate in detail the relationship between breaking of Lorentz symmetry and soliton speed selection mechanism, starting from the minimal model (28).

\subsection{The minimal case}

In the minimal case, the fields, $\Phi$ and $\theta$ do not interact one with the other. This means that the field theory has two completely decoupled sectors. The field equations (29) can be written as follows,

$$
\frac{1}{c_{m}^{2}} \theta_{t t}-\theta_{x x}=-\frac{K}{a+b} \sin \theta, \quad \frac{1}{c_{s}^{2}} \Phi_{t t}-\Phi_{x x}=-\frac{K}{b} \sin \Phi,
$$

where $c_{m}$ is as in Eq. (30). Notice that we have three fundamental speeds in the theory, viz. $c_{m}, c_{t}, c_{s}$ with $c_{m}<c_{t}<c_{s}$. Here $c_{m}$ and $c_{s}$ are the maximal speeds of propagation respectively for $\theta$ - and $\Phi$-waves. The physical meaning of $c_{t}$ will be clear later.

Because of the decoupling, the two sectors of the field theory are invariant under two different groups of Lorentz transformations. Using a notation in which the dependence of a boost from the upper bound speed is evident, we will denote with $\Gamma\left(c_{0}\right)$ the boost in (33). Whereas the field equation (35) for $\theta$ is invariant under boosts $\Gamma\left(c_{m}\right)$, that for $\Phi$ is invariant under $\Gamma\left(c_{s}\right)$. The 
previous symmetry properties have a simple physical consequence: the existence of solitonic excitation for the fields $\theta$ and $\Phi$ which propagate at two independent speeds $\bar{v}, v$ (see section 5 ). The $\Phi$-soliton, $\Phi(x+v t)$ can be obtained boosting the static soliton $\Phi(x)$ with $\Gamma\left(c_{s}\right)$, whereas the $\theta$-soliton, $\theta(x+\bar{v} t)$ can be obtained boosting the static soliton $\theta(x)$ with $\Gamma\left(c_{m}\right)$. The solutions can be written in an Lorentz invariant form introducing the covariant vectors

$$
k^{\mu}=(k, \omega), \quad \bar{k}^{\mu}=(\bar{k}, \bar{\omega}), \quad x^{\mu}=\left(c_{s} t, x\right), \quad \bar{x}^{\mu}=\left(c_{m} t, x\right) .
$$

Propagating solutions are function of the Lorentz scalars,

$$
\Phi=\Phi\left(k_{\mu} x^{\mu}, k_{\mu} k^{\mu}\right) \quad, \quad \theta=\theta\left(\bar{k}_{\mu} \bar{x}^{\mu}, \bar{k}_{\mu} \bar{k}^{\mu}\right)
$$

The condition $\theta=\Phi$ couples the two otherwise decoupled sectors and breaks Lorentz invariance. In fact $\theta=\Phi$ requires $v=\bar{v}$, whereas Lorentz invariance would require $\Gamma\left(c_{s}\right)=\Gamma\left(c_{m}\right)$, which is manifestly impossible for $c_{s} \neq c_{m}$. As shown in Section 5. the condition $\theta=\Phi$ fixes the speed of the soliton so that we have $v=\bar{v}=c_{t}$. The soliton propagating at this speed cannot be obtained by boosting a static solution. This is also evident considering that Eqs. (31) do not allow for static solutions with $\Phi=\theta$.

From a physical point of view the mechanism described above can be seen as a speed selection effect generated by breaking of the Lorentz symmetry. We have a media with two different maximal propagation speeds, $c_{s}, c_{m}$ that can be identified as the propagation speed of $\Phi$ - and $\theta$-sound waves. Furthermore, we have a constraint, $\Phi=\theta$ which breaks the Lorentz symmetry and singles out a solitonic perturbation which can propagate only at the fixed speed $c_{t}$.

\subsection{Interacting models}

Until now we have considered only the minimal, non interacting model (28). Passing to consider the interacting model (24) there is only one main difference: as now the fields $\Phi, \theta$ are coupled to each other, only one single boost $\Gamma\left(c_{s}\right)$ can be defined, and the Lorentz symmetry is broken already at level of the action (24). Being mutually coupled, travelling wave solutions for $\theta, \Phi$ now have to propagate with the same velocity $v$. Using the covariant notation of Eq. (36) they take the form

$$
\Phi=\Phi\left(k_{\mu} x^{\mu}, k_{\mu} k^{\mu}, \omega^{2} c_{s}^{2} / c_{m}^{2}-k^{2}\right), \quad \theta=\theta\left(k_{\mu} x^{\mu}, k_{\mu} k^{\mu}, \omega^{2} c_{s}^{2} / c_{m}^{2}-k^{2}\right) .
$$

Owing to the dependence on the non-Lorentz invariant term $\left(\omega^{2} c_{s}^{2} / c_{m}^{2}-k^{2}\right)$ the solutions are not Lorentz-invariant. Although the boosts $\Gamma\left(c_{s}\right)$ are not a symmetry of the Lagrangian (24) they can be used as solution-generating transformations. A solitonic solution travelling with speed $v$ can be obtained boosting a static solution. Similarly to what happens in the minimal case, the condition $\theta=\Phi$ breaks this solution-generating symmetry. The $\theta=\Phi$ solution cannot be generated by boosting a static solution satisfying the constraint, so that the velocity $v=c_{t}$ for the soliton is selected. 


\section{Discrete symmetries and topological classifi- cation}

The internal (discrete) symmetries of our model are very important both for understanding the peculiarities of the fixed-speed $\theta=\Phi$ solution and for the topological classification of the solitons.

We are dealing with a 2D field theory with broken Lorentz symmetry, for which the internal symmetry group of the Lagrangian (24) is not necessarily the same as that of the reduced action (25) describing travelling wave solutions. We are only interested in the symmetries relevant for the solitonic solutions, namely in the symmetries of the reduced Lagrangian (25).

For $\widetilde{J} \neq 0$ and for generic coupling functions $F, G$ the internal symmetry group of the model - denoted as $\mathcal{G}$ in the following - and the residual symmetry of the vacua - denoted as $\mathcal{H}$ below - are the same as those of the simple SG system (32). We have $\mathcal{G}=\mathbf{Z} \times \mathbf{Z}_{2}$, where $\mathbf{Z}$ is realized as discrete translations $\Phi \rightarrow \Phi+2 \pi n, \theta \rightarrow \theta+2 \pi n$, whereas $\mathbf{Z}_{2}$ is the inversion $\theta \rightarrow-\theta, \Phi \rightarrow-\Phi$. The vacua $\Phi=\theta=2 m \pi$ are invariant under an inversion followed by a translation of $4 \pi m$. The residual symmetry group of the vacua is therefore $\mathcal{H}=\mathbf{Z}_{2}$.

For $\widetilde{J}=0$ the group $\mathcal{G}$ acquires an additional $\mathbf{Z}_{2}$ factor: $\mathcal{G}=\mathbf{Z} \times \mathbf{Z}_{2} \times \mathbf{Z}_{2}$. This third factor comes from the invariance of the Lagrangian (25) under the transformation

$$
\theta \longleftrightarrow \Phi
$$

The group $\mathcal{H}$ acquires an additional factor as well: $\mathcal{H}=\mathbf{Z}_{2} \times \mathbf{Z}_{2}$. Notice that the speed-selecting constraint leaves invariant both $\mathcal{G}$ and $\mathcal{H}$. In fact the submanifold $\Phi=\theta$ is selected by the constraint.

The solitonic solutions of the model can be therefore classified in two classes, which are mapped one into the other by the transformation (38). The solitonic solutions with $\theta=\Phi$ are self-dual, i.e a fixed point of the transformation (38).

The previous discussion holds true for generic coupling functions $F, G$. For particular choices of $F$ and $G$ we can have bigger $\mathcal{G}$ and $\mathcal{H}$ groups. This happens for instance when $F$ and $G$ are periodic functions. In this case $\mathcal{G}$ is the product of two $\mathbf{Z}$ groups times some $\mathbf{Z}_{2}$ factors. However, the $\theta=\Phi$ condition breaks invariance under one of the two $\mathbf{Z}$ groups and we are left with the same symmetry groups $\mathcal{G}$ and $\mathcal{H}$ as above.

The discrete symmetries of SG-like field theories are also of fundamental importance for the topological classification of the solitonic excitations of the theory. The admissible number of solitons of the SG model is determined by the number of ways in which the points $x= \pm \infty$ can be mapped into the different vacua of the model.

We have therefore a one-to-one correspondence between solitons and the elements of the homotopy group $\pi_{0}(\mathcal{G} / \mathcal{H})$. It is also well-known that field theories that admit topological solitonic solutions have conserved currents corresponding to conserved topological charges, which can be identified with the winding numbers of the soliton. 
Using the previous results one can easily calculate the homotopy group $\pi_{0}$ in the case of generic coupling functions $F, G$. For $\widetilde{J} \neq 0$ this is given by

$$
\pi_{0}(\mathcal{G} / \mathcal{H})=\pi_{0}\left(\mathbf{Z} \times \mathbf{Z}_{2}\right) / \mathbf{Z}_{2}=\mathbf{Z} .
$$

The same result holds true when $\widetilde{J} \neq 0$ :

$$
\pi_{0}(\mathcal{G} / \mathcal{H})=\pi_{0}\left(\mathbf{Z} \times \mathbf{Z}_{2} \times \mathbf{Z}_{2}\right) /\left(\mathbf{Z}_{2} \times \mathbf{Z}_{2}\right)=\mathbf{Z} .
$$

As expected the solitonic solution of the model can be labeled by an integer $n$, its topological charge.

For periodic coupling functions the homotopy group $\pi_{0}$ of the model becomes $\mathbf{Z} \times \mathbf{Z}$ and we have two topological charges $(n, m)$ associated to the soliton. However, the condition $\theta=\Phi$ reduces the symmetry group and we are left again with $\pi_{0}=\mathbf{Z}$ and a single topological charge $n$.

\section{$8 \quad$ Stability}

The stability of the solutions we have considered in the previous section is a crucial requirement for our speed selection mechanism. First, being the dynamics non linear we expect high sensitivity of the solutions to the initial conditions. If the $\theta=\Phi$ solution is not stable, small perturbations in the initial conditions may disrupt the mechanism. Second, even though a soliton with the given speed is generated, it may be unstable under perturbation and decay in a finite time.

In the previous section we have seen that the fixed-speed solitonic SG soliton (6), although resulting from breaking of the Lorentz symmetry, share many feature with the usual SG solitons: they are localized, finite energy solutions of the same ordinary differential equation, they allow for the same topological classification and associated topological charges. These features strongly suggest that the fixed-speed SG solitons we are discussing in this paper are stable solutions, in the same way as relativistic SG solitons are.

The analysis of the stability of the solutions of the model (24) is rather complicate. In this paper we will restrict our investigations to the classical stability of the speed-selecting solution $\theta=\Phi$ under linear perturbations. To begin with, we will briefly summarize well-known results about the classical stability of the Solitons of the simple SG model (32).

To discuss the stability one first uses Lorentz invariance to chose the reference frame in which the soliton (34) is static and then considers small perturbations around the static solution,

$$
\varphi(x, t)=\varphi_{0}(x)+\psi(x, t), \quad \psi<<1 .
$$

Inserting this position in the field equations, considering only the linear approximation and separating the variables in the perturbation, i.e setting $\psi(x, t)=$ $\Psi(x) e^{i \omega t}$, one obtains an equation for $\Psi$, which has the form of a time-independent Schrodinger equation

$$
T \Psi=\left(\frac{\omega}{c_{0}}\right)^{2} \Psi, \quad T=-\frac{d^{2}}{d x^{2}}+\omega_{0}^{2}\left(1-2 \operatorname{sech}^{2} x\right) .
$$


Classical stability requires the eigenvalues of the operator $T$ to be real and nonnegative. This is guaranteed if the operator $T$ is self-adjoint and non-negative. These two properties of the operator $T$ given in Eq. (40) can be shown in different ways.

The most elegant way is to use supersymmetric (SUSY) factorization 24, 35, 36. One can find a superpotential $W(x)$ such that $T=A^{+} A$ with $A=$ $(d / d x)-W(x), A^{+}=-(d / d x)-W(x)$, from which immediately follows that $T$ is self-adjoint, whereas the SUSY algebra implies that the spectrum of $T$ is non-negative. Being $T$ a SUSY Hamiltonian, the Schrodinger equation (40) can be explicitly solved. It turns out that the spectrum has a single discrete mode $\psi_{0}$ for $\omega=0$, and a continuum part $\psi_{k}$ for $\omega>\omega_{0} c_{0}$ [4].

The discrete mode corresponds to soliton translations, whereas the continuos modes are not reflected by the potential in Eq. (40) and describe soliton deformations. The set $\left\{\psi_{0}, \psi_{k}\right\}$ is a complete, orthonormal basis and can be therefore used to expand a generic perturbation $\psi(x, t)$.

Let us now consider our generalized SG model (24). To simplify the calculation we will first consider a vanishing coupling function $F$, later we will discuss the most general case. For $F=0$, introducing the new dimensionless variables $t^{\prime}=\omega_{0} c_{s} t$ and $x^{\prime}=\omega_{0} x$, with $\omega_{0}=K / b$ and the dimensionless parameter $\alpha=a / b$, the field equations stemming from the Lagrangian (24) are

$$
\begin{aligned}
& \Phi_{t^{\prime} t^{\prime}}-\Phi_{x^{\prime} x^{\prime}}=-\sin \Phi+\frac{1}{\omega_{0}^{2}} \frac{\partial G}{\partial \Phi} \\
& \left(1+\alpha \frac{c_{s}^{2}}{c_{t}^{2}}\right) \theta_{t^{\prime} t^{\prime}}-(1+\alpha) \theta_{x^{\prime} x^{\prime}}=-\sin \theta+\frac{1}{\omega_{0}^{2}} \frac{\partial G}{\partial \theta} .
\end{aligned}
$$

We want to investigate the classical stability of the $\theta=\Phi$ soliton solution of the previous equations, which propagates at fixed speed $v=c_{t}$. It is therefore appropriate to choose a frame in which this soliton is at rest. This can be easily done by performing in Eqs. (41) a boost of speed $c_{t}$,

$$
y=\gamma\left(x^{\prime}+\frac{c_{t}}{c_{s}} t^{\prime}\right), \quad \tau=\gamma\left(t^{\prime}+\frac{c_{t}}{c_{s}} x^{\prime}\right), \quad \gamma=\frac{1}{\sqrt{1-\left(\frac{c_{t}}{c_{s}}\right)^{2}}} .
$$

In the $(\tau, y)$ frame, Eqs. (41) read

$$
\begin{aligned}
& \Phi_{\tau \tau}-\Phi_{y y}=-\sin \Phi+\frac{1}{\omega_{0}^{2}} \frac{\partial G}{\partial \Phi} \\
& \theta_{\tau \tau}-\theta_{y y}+\alpha\left(1+\frac{c_{s}^{2}}{c_{t}^{2}}\right) \theta_{\tau \tau}+2 \alpha \frac{c_{s}}{c_{t}} \theta_{\tau} \theta_{y}=-\sin \theta+\frac{1}{\omega_{0}^{2}} \frac{\partial G}{\partial \theta} .
\end{aligned}
$$

Notice that the third and fourth term of the l.h.s. of the second equation are responsible for the breaking of Lorentz symmetry. Seen in the $(\tau, y)$ frame, the solitonic solution of fixed speed $c_{t}$ is static; it is given by

$$
\Phi_{0}(y)=\theta_{0}(y)=\arctan e^{y} .
$$


The classical stability of the previous solution is investigated by considering small perturbations $\sigma, \chi$ around the static solution,

$$
\Phi(y, \tau)=\theta_{0}(y)+\sigma(y, \tau), \quad \Theta(y, \tau)=\theta_{0}(y)+\chi(y, \tau) .
$$

At first order in the perturbation Eqs. (43) give,

$$
\begin{aligned}
& \sigma_{\tau \tau}-\sigma_{y y}=-\left(\cos \theta_{0}\right) \sigma+B(\sigma-\chi) \\
& \chi_{\tau \tau}-\chi_{y y}+\alpha\left(1+\frac{c_{s}^{2}}{c_{t}^{2}}\right) \chi_{\tau \tau}+2 \alpha \frac{c_{s}}{c_{t}} \theta_{\tau}\left(\theta_{0}\right)_{y}=-\sin \theta-B(\sigma-\chi),
\end{aligned}
$$

where $B$ is a constant given by $B=\left(1 / \omega_{0}^{2}\right)\left(\partial^{2} G / \partial \Phi^{2}\right)(0)$.

For generic values of the parameter $\alpha$, the system (46) is very hard to solve. For $\alpha<<1$ we can treat the terms proportional to $\alpha$ as a perturbation. At zeroth order in the perturbative expansion we can neglect them. In this case we can write Eqs. (46) in operator form, introducing the Schrodinger-like operator $R$ :

$$
R\left(\begin{array}{c}
\sigma \\
\chi
\end{array}\right)=-\frac{\partial^{2}}{\partial \tau}\left(\begin{array}{l}
\sigma \\
\chi
\end{array}\right) .
$$

The operator $R$ can be diagonalized by the linear transformation $\varphi_{1}=\sigma-\chi$, $\varphi_{2}=\sigma+\chi$

$$
R=\left(\begin{array}{cc}
-\frac{\partial^{2}}{\partial y^{2}}+V_{1}(y) & 0 \\
0 & -\frac{\partial^{2}}{\partial y^{2}}+V_{2}(y)
\end{array}\right) .
$$

where the potentials $V_{1}$ and $V_{2}$ are

$$
V_{1}=\left(1-2 \operatorname{sech}^{2} y\right), \quad V_{2}=\left(1+2 B-2 \operatorname{sech}^{2} y\right) .
$$

Separating the variables in the perturbations, i.e setting $\varphi_{1}(y, \tau)=\varphi_{1}(y) e^{i \omega_{1} \tau}$, $\varphi_{2}(y, \tau)=\varphi_{2}(y) e^{i \omega_{2} \tau}$, equation (47) becomes a time-independent Schrodingerlike equation.

There are two particular values of the parameter $B$ which correspond to SUSY models, $B=0$ and $B=3 / 2$. In both cases the operator $R$ is self-adjoint and non-negative, implying stability of the background solution. The $B=0$ case corresponds to the minimal, decoupled case discussed in Sect. 5. The diagonal elements $R_{1}$ and $R_{2}$ of the operator $R$ of Eq. (48) corresponding respectively to the perturbations $\varphi_{1}, \varphi_{2}$, are equal and coincide with the previously discussed operator $T$ of Eq. (40). The superpotential is also the same and is given by $W_{1}=W_{2}=\tanh y . \quad R_{1}$ and $R_{2}$ have therefore the same spectrum, which coincides with that of the operator $T$.

For $B=3 / 2$, the operators $R_{1}$ and $R_{2}$, although not equal, correspond both to SUSY Hamiltonians. $R_{1}$ is equal to the operator $T$ of Eq. (40) whereas $R_{2}$ correspond to a different SUSY Hamiltonian (see e.g. Ref. [35, 36]). The superpotential is given by $W_{2}=2 \tanh (y)$. The spectrum of $R_{2}$ has two discrete modes with eigenvalues $\omega_{2}=0$ and $\omega_{2}=(3 / 4) \omega_{0} c_{s}$. The continuum part of the spectrum is located at $\omega_{2} \geq 4 \omega_{0} c_{s}$. 
For generic $B$ the operator $R_{1}$, being independent from $B$, corresponds always to the SUSY Hamiltonian discussed above. On the other hand the operator $R_{2}$ does not seem to correspond to a SUSY Hamiltonian. From the general form of the potential $V_{2}$, see Eq. (49), one can easily infer that we have a discrete spectrum for $\omega_{2}<(1+2 B) \omega_{0} c_{s}$, whereas we have a continuum spectrum for $\omega_{2} \geq(1+2 B) \omega_{0} c_{s}$. However, we do not have any argument to show that the eigenvalues of the discrete spectrum of $R_{2}$ are non negative. In the following we will consider only the cases $B=0$, but our considerations can be easily extended to the case $B=3 / 2$.

Let us now consider the first order in the perturbation expansion in the parameter $\alpha$, i.e let us consider the system in the complete form (46). For $B=0$ the first equation of the system can be written as

$$
\sigma_{\tau \tau}-\sigma_{y y}=-\left(1-2 \operatorname{sech}^{2}(y) \sigma .\right.
$$

Comparing with Eq. (40), we immediately realize that this corresponds to the operator $T=R_{1}$. It follows immediately that our fixed speed solitonic solution (44) is stable for small perturbation of the field $\Phi$. After some manipulations the equation for the perturbation $\chi$ in (46) (and $B=0$ ) can be written as

$$
C \chi_{\tau \tau}-\chi_{y y}+D(\operatorname{sech} y) \chi_{\tau}+\left(1-2 \operatorname{sech}^{2} y\right) \chi=0,
$$

where $C=1+\alpha\left[1+\left(c_{s} / c_{t}\right)^{2}\right], D=\alpha\left(c_{s} / c_{t}\right)$. The previous equation differs from the equation (501) in two points. First, the term $\chi_{\tau \tau}$ is multiplied by $C$. This is rather harmless, it just means that the eigenvalues of the operator $T$ have to be rescaled by the positive factor $C$. This term cannot change the semi-positivity of the eigenvalues. Second, there is an $y$-dependent dissipative term. This term becomes negligible for $y \rightarrow \pm \infty$. In fact in this limit Eq. (51) gives

$$
C \chi_{\tau \tau}-\chi_{y y}+\chi=0
$$

which has normal mode solutions $\chi=A e^{i(k y+\omega \tau)}$ with dispersion relation $C \omega^{2}=$ $k^{2}+1$.

The damping term is maximized near the minimum of the potential $V(y)$, $y=0$. Near $y=0$ the perturbation $\chi$ has a damping term $\chi \propto e^{-\beta \tau}$, where $\beta$ is some constant. It is interesting to notice in this context that the Lorentz symmetry breaking terms act on the perturbation as damping term and as rescaling of the eigenvalues of the operator $T$.

The equation (51) can be solved by expanding $\chi$ in terms of the eigenfunctions $\left\{\psi_{0}, \psi_{k}\right\}$ of the operator $T$ which represent a complete orthonormal set,

$$
\chi(y, \tau)=\psi_{0}(y) h(\tau)+\int_{-\infty}^{\infty} \psi_{k}(y) g_{k}(\tau) d k .
$$

Inserting this equation into (51), using the orthonormal character relations, and the fact that $\left\{\psi_{0}, \psi_{k}\right\}$ are eigenfunction of the operator $T$ corresponding to eigenvalues 0 and $\omega^{2}$, one obtains after some manipulations the equations for $h$ 
and $g_{k}$; these turn out to be

$$
\begin{aligned}
& C \frac{d^{2} h}{d \tau^{2}}+2 \pi D \frac{d h}{d \tau}=0 \\
& C \frac{d^{2} g_{l}}{d \tau^{2}}+D \int_{-\infty}^{\infty} d k d y\left[\operatorname{sech}(y) \psi_{l}^{*} \psi_{k} \frac{d g_{k}}{d \tau}\right]+\omega^{2} g_{l}=0
\end{aligned}
$$

The equation for $h$ is immediately solved to give

$$
h(\tau)=P e^{-\beta \tau}+Q
$$

where $P, Q$ are integration constants and $\beta=2 \pi D / C$. From Eq. (54) it follows that after a transition time, only the pure translation mode $\psi_{0}$ of the SG soliton will survive (when $Q \neq 0$ ); or that this mode will be completely damped to zero and only the static background SG solution will survive (for $Q=0$ ). In both cases, this shows stability of our fixed speed soliton under perturbations of the discrete spectrum.

The equation for the functions $g_{l}(\tau)$ in Eq. (53) is more involved. It is a integro-differential equation that cannot be solved in closed form. However, from the general structure of the differential equation, it is evident that the solutions will always have an oscillatory part and a damping term $\sim e^{-\beta_{l} \tau}$.

Qualitatively the behavior of $g$ will be therefore similar to the previously described one for $h$. After a transition time, the normal modes corresponding to the continuum spectrum of the operator $T$ will be either completely damped or will survive as simple soliton deformations. This provides strong arguments for the stability of our solitonic solution for perturbations of the continuum spectrum.

Until now we have considered explicitly stability when the coupling function $F$ vanishes. Our discussion can be easily extended to the case when $F \neq 0$. Looking at the Lagrangian (24) one easily realizes that the terms introduced in this way are invariant under Lorentz transformations $\Gamma\left(c_{s}\right)$. In the perturbation approach these terms have two effects. They change the value of the parameter $C$ in Eq. (51), and introduce a term proportional to $\operatorname{sech}^{2} y(\sigma-\chi)$ in both Eqs. (46). The modification of $C$ does not change the qualitative outcome of our previous discussion, whereas the modified Eqs. (46) can be analyzed using the same method we have previously used.

\section{Conclusions}

In this paper we have proposed a mechanism for fixing the velocity of relativistic solitons. The proposal has been first elaborated for a molecular chain model of double coupled pendulums. It has been then generalized to a full class of $2 \mathrm{D}$ field theories of the sine-Gordon type.

From a phenomenological point of view, the model allows one to select the speed of a SG soliton just by tuning the elastic coupling constants and the kinematical parameters that support the transverse sound wave. Thus, the 
selection speed mechanism could be at work whenever SG solitons appear with a given speed - and could be useful to formulate models whenever one needs to explain phenomena of this type, or however where the soliton speed plays a special role and should be tuned. Moreover, our selection mechanism could be also used to devise and realize non-linear media where SG solitons travel at a given fixed speed.

Our results are also relevant from a fundamental, field-theoretical point of view. In fact we have shown that the speed selection mechanism is deeply related both with the existence of some conditionally conserved quantity and to the breaking of the Lorentz symmetry of the usual SG models. The most striking and highly non trivial result we have obtained in this context is that the characterizing features of relativistic SG solitons - that is, finiteness and localization of energy, topological classification and stability - may be still preserved even if the Lorentz symmetry is broken and a soliton of a given speed is selected.

We have shown that the solitonic excitation with fixed speed of our model are classically stable and have topological conserved charges. In particular it turns out that the Lorentz symmetry breaking terms act on the perturbations around the fixed speed soliton as a damping term.

This result is particularly intriguing in view of the analogy between relativistic particles and SG solitons. It can be seen as a strong indication that relativistic nature of the particle is not a necessary condition for the analogy to hold. In particular one can speculate about the existence of SG solitons which behave as non-relativistic (e.g Newtonian) particles.

\section{References}

[1] J. Scott Russell, "Report on waves", Proc. of the British Association for the Advancement of Science, London, (1845).

[2] D. J. Korteweg and G. de Vries, Phil. Mag. 39, 422 (1895).

[3] N. J. Zabusky and M. D. Kruskal, Phys. Rev. Lett. 15, 240 (1965).

[4] Th. Dauxois and M. Peyrard, Physique de solitons, Editions CNRS (Paris) 2004; Physics of Solitons, Cambridge UP (Cambridge) 2006.

[5] R.K. Dodd, J. C. Eilbeck, J. D. Gibbon, H.C. Morris, Solitons and Nonlinear Wave Equations, Academic Press, London, New York (1982).

[6] J. Rubinstein, J. Math. Phys. 11, 258 (1970).

[7] A.S. Davydov, Solitons in Molecular Systems, Kluwer (Dordrecht) 1981.

[8] S.W. Englander, N.R. Kallenbach, A.J. Heeger, J.A. Krumhansl and A. Litwin, Proc. Nat. Acad. Sci. USA 77 (1980), 7222-7226.

[9] L.V. Yakushevich, Nonlinear Physics of DNA, Wiley (Chichester) 1998; second edition 2004. 
[10] G. Gaeta, C. Reiss, M. Peyrard and Th. Dauxois, Rivista del Nuovo Cimento 17 (1994) n.4, 1-48.

[11] M. Peyrard, Nonlinearity 17 R1-R40 (2004).

[12] A. Barone, G. Paternó, Physics and applications of the Josephson effect, Wiley, New York (1982).

[13] E. Feldtkeller, Phys. Stat. Sol. 27, 161 (1968).

[14] V.G. Bar'yakhtar, Dynamics of topological magnetic solitons, Springer Tracts in Mod. Phys., Springer, Berlin (1994).

[15] G. L. Lamb, Rev. Mod. Phys. 43, 99 (1971)

[16] J. R. Taylor, Optical Solitons, Cambridge University Press, Cambridge (1992.

[17] C. Cattuto, F. Marchesoni, Phys. Rev. Lett. 79, 5070 (1997).

[18] N. Manton, and P. Sutcliffe, Topological solitons, Cambridge University Press, Cambridge (2004).

[19] G. L. Lamb, Elements of Soliton Theory, (Wiley, New York) (1980).

[20] D. Finkelstein and C. W. Misner, Ann. Phys. 6, 230 (1969).

[21] T.H.R. Skyrme, Proc. Roy. Soc. London A 247, 260 (1958).

[22] A.B. Zamolodchikov and A.B. Zamolodchikov Ann. Phys. 120253 (1979)

[23] E. Witten, Nucl. Phys. B223, 433 (1983).

[24] E. Witten, Nucl. Phys. B185, 513 (1981).

[25] J. Gegenberg and G. Kunstatter, Phys. Lett. B $4 \mathbf{4 1 3} 274$ (1997) arXiv:hep-th/9707181.

[26] M. Cadoni, Phys. Rev. D 58, 104001 (1998) arXiv:hep-th/9803257.

[27] F. Calogero and A. Degasperis, Spectral transform and solitons, North Holland (Amsterdam) 1982.

[28] V. Zakharov, What is integrability? Springer, Berlin 1991.

[29] A. Dubrovin, S.P. Novikov and A. Fomenko, Modern geometry (voll. I, II \& III), Springer (Berlin) 1984.

[30] M. Cadoni, R. De Leo and G. Gaeta, q-bio.BM/0604014 (In press in Phys. Rev. E).

[31] M. Cadoni, R. De Leo and G. Gaeta, q-bio.BM/0604027 (In press in J. Nonlinear Math. Phys.). 
[32] M. Peyrard, B. Piette and W. J. Zakrzewski, Physica D 64, 355 (1993).

[33] E. Harikumar, C. Nagaraja Kumar, and M. Sivakumar, Phys. Rev. D 58, 107703 (1998).

[34] V. W. Sarlet, P.G.L. Leach and F. Cantrijn, Physica D 17, 87 (1985).

[35] F. Cooper, A. Khare, U. Sukhatme, Phys. Rep. 251, 267 (1995).

[36] F. Cooper, A. Khare, U. Sukhatme, Supersymmetry in Quantum Mechanics, World Scientific, Singapore (2001). 